
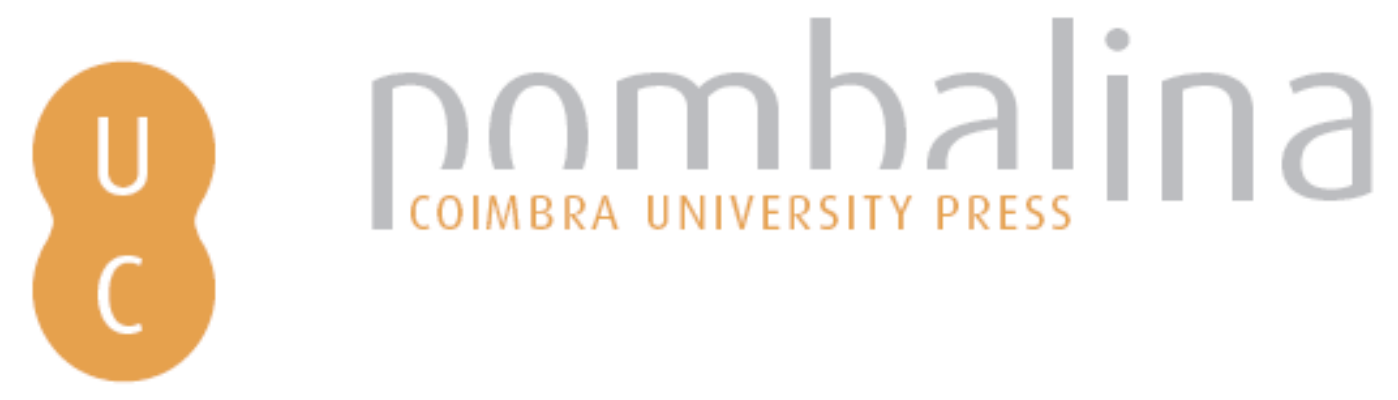

Veneno simposíaco: envenenamiento en los banquetes en la obra plutarquea

Autor(es): $\quad$ Romero González, Dámaris

Publicado por: Imprensa da Universidade de Coimbra; Centro de Estudos Clássicos e

URL

persistente: URI:http://hdl.handle.net/10316.2/32003

DOI: $\quad$ DOI:http://dx.doi.org/10.14195/978-989-8281-17-3_23

Accessed : $\quad$ 26-Apr-2023 12:11:12

A navegação consulta e descarregamento dos títulos inseridos nas Bibliotecas Digitais UC Digitalis, UC Pombalina e UC Impactum, pressupõem a aceitação plena e sem reservas dos Termos e Condições de Uso destas Bibliotecas Digitais, disponíveis em https://digitalis.uc.pt/pt-pt/termos.

Conforme exposto nos referidos Termos e Condições de Uso, o descarregamento de títulos de acesso restrito requer uma licença válida de autorização devendo o utilizador aceder ao(s) documento(s) a partir de um endereço de IP da instituição detentora da supramencionada licença.

Ao utilizador é apenas permitido o descarregamento para uso pessoal, pelo que o emprego do(s) título(s) descarregado(s) para outro fim, designadamente comercial, carece de autorização do respetivo autor ou editor da obra.

Na medida em que todas as obras da UC Digitalis se encontram protegidas pelo Código do Direito de Autor e Direitos Conexos e demais legislação aplicável, toda a cópia, parcial ou total, deste documento, nos casos em que é legalmente admitida, deverá conter ou fazer-se acompanhar por este aviso.

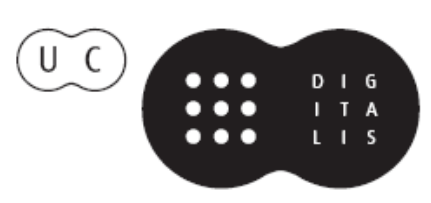




\section{Symposion and Philanthropia in Plutarch}

\section{José Ribeiro Ferreira, Delfim Leão Manuel Troster e Paula Barata Dias (eds.)}

IMPRENSA DA UNIVERSIDADE DE COIMBRA 


\title{
VENENO SIMPOSÍACO: ENVENENAMIENTO EN LOS BANQUETES EN LA OBRA PLUTARQUEA ${ }^{\mathrm{I}}$
}

\author{
DÁmaris Romero GonzÁlez \\ Universidad de Birmingham
}

\begin{abstract}
In the festive environment of the banquet, it is possible to identify moments when this mood has been broken, and Plutarch presents many examples of it in the Vitae and Moralia. One possibility of disrupting this context is poisoning the guest, as Parysatis killed Stateira, or trying to do it, such as Medea did with Theseus or Neoptolomeus with Phyrrus. Apart from relating these deaths, or failed attempts, by poison at the banquet, Plutarch also adds the consequences for the hosts of breaking down this happy time and the reasons why they committed the murders.
\end{abstract}

Plutarco, consciente de que el envenenamiento era un crimen bastante habitual, presenta numerosos ejemplos de esto en Vidas y, en menor medida, en Moralia $^{2}$. Ahora bien, esto no siempre tiene lugar en los banquetes y como muestra de ello se encuentran los siguientes ejemplos, que, por no estar encuadrados dentro del contexto simposíaco, quedan descartados: un primer grupo son los envenenamientos por error de una persona ${ }^{3}$; un segundo, los que se producen por la mezcla del veneno con un líquido ${ }^{4}$ o con un alimento sólido ${ }^{5} \mathrm{y}$, finalmente, un tercero, en los que el queronense no da ninguna pista que induzca a pensar que se realizan en un banquete . $^{6}$

\section{Del complot al envenenamiento}

La imagen que se tiene del banquete griego es, en general, idílica: se presenta como la reunión en un ambiente amigable de un grupo limitado de

${ }^{1}$ Agradezco a la Fundación Hardt (Ginebra) la beca que me concedió para consultar su biblioteca y poder realizar esta comunicación. Parto como base del artículo de Rosa M. ${ }^{\mathrm{a}}$ Aguilar, 2008, en el que se estudia la frecuencia y los sentidos de la palabra pharmakon en el queronense.

${ }^{2}$ Como R. M. a Agullar, 2008, pp. 754-5, 759 dice "en general, excepto si se trata de alguna anécdota, el significado (en Mor.) es el de remedio o medicamento, bien en sentido recto o en el figurado. (...) De otra parte, el reparto de significados resulta coherente con el tipo de discurso. En los textos históricos el significado de 'veneno' aparece porque el $\varphi \alpha ́ \alpha \mu \alpha \kappa o v ~ s e$ usa para ejecutar al enemigo o eliminarle ocultamente o, por fin, en un suicidio como recurso último ante la derrota. En cambio, en textos de carácter discursivo o filosófico el significado de 'medicamento' o 'remedio' muestra mayor frecuencia y, las más veces, figuradamente".

${ }^{3}$ Lúculo, quien murió por los supuestos filtros amorosos de su esclavo (Luc. 43.1-2).

${ }^{4}$ Sinorix a manos de Camma ante el altar de Ártemis (Mor. 258B-C, 768C (la mezcla de leche y miel - o también hidromiel).

${ }^{5}$ Creso, aunque, gracias a la lealtad del panadero, no llegó a comer el pan envenenado que su madrastra quería darle (Mor. $401 \mathrm{E}-\mathrm{F})$; el asesinato de un padre por un hijo en un pastel (Cic. 26.5).

${ }^{6} \mathrm{El}$ intento de asesinato de Aretafila a su marido Nicócrates y descubierto por su suegra (Mor. 256 B-D), el de Hirodes por su hijo Fraates (Crass. 33,5) o el de Pirro por su médico (Pyrrh. 21.1, Mor. 195B), Elisio (Mor. 109B), los invitados del padre de Tespesio (Mor. 566F). 
personas, con una determinada vinculación entre ellos, que se reunían en salas privadas de una casa ${ }^{7}$, acompañados de ciertos divertimentos, como el disfrute del vino y la comida, la recitación o la conversación, entre otros ${ }^{8}$. De hecho, en los ejemplos que dan origen a esta comunicación se cumplen gran parte de estos requisitos: se evidencia el tipo de vinculación que unía a los asistentes, como la familiaridad en el caso de Parisatis y Estatira, suegra y nuera respectivamente; la política, así Gelón busca en Mírtilo un aliado "político" para Neoptólemo; y la hospitalidad, cuando Teseo es agasajado como huésped de Egeo. También se disfruta del vino y la comida, ya sea como divertimento de los convidados, sirviendo como ejemplo los brindis que hubo en el banquete de Gelón o la comida dispuesta para agasajo de Teseo, ya sea como instrumento de muerte, así Teseo lleva el veneno en su copa y Estatira muere por el ave ryntakis envenenado ofrecido por Parisatis.

Si bien es cierto que la mayoría de las veces el ambiente suele ser afable, no significa que siempre sea así. Como dice F. B. Titchener, "un banquete era un lugar sorprendentemente peligroso para estar, por norma general": los banquetes son propicios para los atentados o para planearlos. Plutarco, conocedor de esta realidad, presenta tres ejemplos en Vidas de envenenamiento en el banquete, que permiten seguir el recorrido del veneno desde los momentos iniciales, en los cuales se urde el complot, hasta su finalización con la muerte de la víctima elegida.

El primer paso para acabar con el adversario es tramar su muerte, en estos casos, usando veneno. Plutarco lo ejemplifica en Pyrrh. 5.7-14. Gelón, partidario de Neoptólemo, encuentra una buena oportunidad de colaborar en la muerte de su enemigo (Pirro) con el desplante que Pirro le hizo a su copero Mírtilo:

(scil. Gelón) le (scil. Mírtilo) invitó a un banquete en el que, como algunos dicen, aparte de aprovecharse entre copas de la juventud de Mírtilo, le estuvo aduciendo razones y exhortándole a que se uniera a Neoptólemo y asesinara a Pirro mediante un veneno ${ }^{10}$.

Ideada la maquinación, ya sólo queda llevarla a cabo. Sin embargo, el tener todos los movimientos planificados, no asegura el éxito, pues siempre hay que considerar el elemento sorpresa. Plutarco lo muestra con los fracasos de Pirro y Teseo.

En el primero de los envenenamientos fracasados, el de Pirro (Pyrrh. 5.10-14), dos fueron las causas por las que no tuvo lugar el emponzoñamiento. Éstas fueron, por una parte, la lealtad de Mírtilo a Pirro, al que le descubrió todo lo que Gelón tramaba, y, por otra, la excesiva confianza de Neoptólemo en la aparente buena marcha del plan, de modo que bajó la guardia y comenzó a hablar abiertamente de la asechanza.

\footnotetext{
${ }^{7}$ P. Garnsey, 1999, p.131.

${ }^{8}$ E. Pellizer, 1994, bosqueja los tipos de entretenimiento en el simposium.

${ }^{9}$ F. B. Titchener, 1999, p. 492.

${ }^{10}$ Pyrrh. 5.8.
} 
El segundo de los envenenamientos fracasados es quizá uno de los más conocidos en la literatura griega y es el de Teseo por parte de Medea en el banquete en que éste se dio a conocer como hijo de Egeo.

(scil. Medea) lo persuadió (scil. a Egeo) para que, invitando al extranjero, lo envenenara. Así pues, yendo Teseo al banquete, pensó que sería mejor no descubrir de primeras quién era, sino que quiso dar una pista para ser descubierto, y, cuando la carne se sirvió, sacando la espada para cortarla, se la enseñó. $\mathrm{Al}$ instante Egeo se percató y dejó caer la copa de veneno... ${ }^{11}$

Medea quiso eliminar a Teseo con el inocente beneplácito de Egeo, desconocedor en ese momento de que el extranjero al que homenajeaban era su hijo. A diferencia del rey, Medea sí lo sabía, puesto que había indagado sobre Teseo cuando éste llegó a Atenas, pero temía que fuera una amenaza para su posición, ya que ella le dio a Egeo un hijo, $\mathrm{Medo}^{12}$. Toda esperanza de matar a Teseo se vio frustrada cuando Egeo reconoció como suya la espada que Teseo utilizó para trinchar la carne del banquete, objeto con el que Medea no había contado.

El único ejemplo que Plutarco ofrece de envenenamiento exitoso es el de Estatira por Parisatis (Art. 19.1-5). Ésta, que era la madre del rey Artajerjes, recelaba de Estatira, esposa del mismo, por el gran influjo que estaba ejerciendo sobre su hijo en detrimento de ella, de modo que decide matarla:

(scil. Parisatis) Tenía una esclava fiel llamada Gigis, con gran influencia sobre ella y quien, según Dinón, la ayudó a preparar el veneno, aunque, según Ctesias, fue cómplice involuntaria... Después de un tiempo de sospechas y disensiones, (scil. Parisatis y Estatira) habían empezado otra vez a visitarse y a cenar juntas, aunque comían de los mismos alimentos y servidos por las mismas manos por desconfianza y precaución... Parisatis, según Ctesias, trinchó una de estas aves con un cuchillo untado por un lado con el veneno, con lo que esa parte del ave quedó emponzoñada; mientras ella se llevó a la boca para comérsela la que estaba limpia de veneno, dio a Estatira la emponzoñada... Así pues la mujer (Estatira) murió con grandes dolores y convulsiones...

\section{De la risa a las lágrimas}

Ahora bien, ¿por qué el banquete deja de ser lugar de divertimento para convertirse en lugar de muerte? Porque las intenciones de los anfitriones no son el esparcimiento de sus invitados, sino la muerte de ellos, y se valen del despreocupado contexto festivo en el que se desarrolla el banquete para llevar a cabo sus planes. Ni Teseo, Pirro o Estatira ${ }^{13}$ imaginan las intenciones de

${ }^{11}$ Thes. 12.2-3.

12 Estos acontecimientos no los relata Plutarco, sino otros autores como Apollod. I.9.28, Epit. 1.5-6, D.S. IV.55.4-6 o Paus. II.3.8.

${ }^{13}$ Quizá Estatira podría imaginarlo de Parisatis pues Plutarco cuenta que ambas comían los mismos alimentos servidos por las mismas manos, pero al ver que Paristis degustaba la misma 
sus compañeros de mesa, ya que "las diversiones son terribles para mantener cualquier actitud distante" 14 .

De ese modo, al producirse la ruptura del ambiente lúdico y sagrado en el que se celebra el simposio ${ }^{15}$, se incumple el objetivo por el que éste se festeja y que Plutarco pone en boca de Teón en sus Quaestiones Convivales:

...concederá un sitio sólo a aquellas conversaciones, espectáculos y bromas que cumplan con la finalidad del banquete, y esto sería producir en los presentes, por medio del placer, un robustecimiento o el origen de una amistad; pues el banquete es un entretenimiento con vino que por el encanto acaba en $\operatorname{amistad}^{16}$.

Ninguno de los tres banquetes pretende ese fin, conseguir una nueva amistad o robustecer una ya existente. Gelón, aprovechando el enfado de Mírtilo por el desplante de Pirro, intenta beneficiarse de la cercanía del copero para asesinar a su adversario. Por su parte, Paristatis, que sentía aversión hacia Estatira y estaba celosa del poder que ésta tenía sobre su hijo, reanuda sus relaciones con su nuera para tramar contra ella. Finalmente, Medea, recurriendo a la costumbre griega de la hospitalidad, quiere agasajar a Teseo para envenenarlo.

Sin embargo, este quebrantamiento no queda impune y, como apunta I. Muñoz ${ }^{17}$, quienes han tramado o cometido el envenenamiento, sufren las consecuencias de este acto.

Neoptólemo, creyendo que la asechanza seguía adelante y rebosante de alegría porque pronto vería muerto a su enemigo, acude a la invitación de Pirro a un banquete, sin sospechar que éste conoce por partida doble sus planes, primero por Mírtilo y luego por la mujer del mayoral. En ese banquete Pirro lo mata, adelantándosele y teniendo de su parte a los principales de los epirotas ${ }^{18}$.

Ahora bien, siguiendo la lógica de lo dicho en el párrafo anterior, Pirro también tendría que haber muerto poco después. Sin embargo, la excepción a esta posible regla, como el propio I. Muñoz señala, se incumple cuando el asesinato se comete como solución a una situación tiránica, tal que sucede aquí: "...debido al odio que les inspiraba la forma opresiva y violenta con que Neoptólemo se conducía en su gobierno" ${ }^{19}$.

Tras descubrirse el intento de envenenamiento de Teseo, Medea es expulsada del Ática, junto con su hijo Medo, por $\mathrm{Egeo}^{20}$.

comida que ella, le haría más confiada. Cf. N. Simões Rodrigues, en este mismo volumen, p. 251.

${ }^{14}$ Per. 7.3 .

${ }^{15}$ Cf. I. Muñoz Gallarte, en este mismo volumen, p. 246.

${ }^{16}$ Mor. 621C.

${ }^{17}$ Cf. I. Muñoz Gallarte, en este mismo volumen, p. 251.

${ }^{18}$ J. M. Mossman, 1992, p. 94, al comparar los intentos de asesinato de Alejandro y Pirro escribe: "Donde Alejandro es un rey que responde a las conspiraciones de hombres inferiores a él, Pirro es un jugador más en un juego de intriga”.

${ }^{19}$ Phyrr. 5.2.

${ }^{20}$ Cf. n. 12. 
Tanto Gigis como Parisatis son castigadas por el emponzoñamiento de Estatira. La condena que recibió la esclava fue, como Plutarco recoge, según la ley en Persia: machacar y moler con una piedra su cabeza, colocada en otra piedra ancha, hasta que la cara y la cabeza quedan deshechas. Para la reina madre, la condena fue más leve y dictada por Artajerjes: es exiliada, estando ella de acuerdo, a Babilonia, ciudad que su hijo Artajerjes no volverá a visitar mientras ella estuviese allíi ${ }^{21}$.

\section{Conclusiones: Razones para el Envenenamiento}

Plutarco, en su faceta de biógrafo, ha hecho acopio de sucesos que pertenecen a la vida de los protagonistas de su obra, completando estas semblanzas con anotaciones de carácter moral, de modo que el queronense no se limita a exponer el envenenamiento de un personaje, sino también las razones que condujeron a ello. Esto ocurre en los casos de Teseo y Estatira, porque en el ejemplo de Pirro no se da ninguna razón para el intento de envenenamiento por parte de Neoptólemo y sin embargo sí se dan muchas para su posterior asesinato: la forma de gobernar de Neoptólemo y su conjura. No obstante, éstas son una excusa de la verdadera razón del asesinato de Neoptólemo: la ambición natural de Pirro (Phyrr. 5.1,14).

La primera causa es el miedo a la usurpación del poder, como le ocurría a Egeo. Plutarco cuenta que Egeo, a causa de sus años, estaba "lleno de celos y sospechas, y temiendo cualquier cosa a causa de la facción que entonces estaba en la ciudad"22. Así pues, Egeo, anciano, estaba sometido a la presión de la presencia de pretendientes al trono, de modo que se deja convencer por Medea para, por una parte, tomar fármacos capaces de hacerle engendrar de nuevo $\mathrm{y}$, por otro, usar un veneno para matar al extranjero $\mathrm{Teseo}^{23}$.

La segunda causa es la mezcla del odio y los celos que Parisatis sentía hacia Estatira. El odio viene provocado por una acumulación de actuaciones en clara oposición a Parisatis por parte de Estatira, quien tampoco sufría a su suegra, como eran la acusación de ser la culpable del inicio de la guerra fraticida, la muerte del eunuco que cortó la cabeza y la mano a Ciro muerto y el cambio de decisión de Artajerjes respecto al destino de Clearco ${ }^{24}$. Los celos son reflejo de la impotencia de Parisatis al ver que la influencia sobre su hijo era fruto del respeto y el honor que él tenía hacia ella, mientras que

${ }^{21}$ Art. 19.6

22 Thes. 12.2 .

${ }^{23}$ H. J. Walker, 1995, pp. 87-8, observa que, en tan sólo dos frases yuxtapuestas, Plutarco expresa los dos poderes antagonistas de los fármacos: "Pueden destrozar completamente a un hombre y su casa (que es lo que Medea intenta hacer cuando Teseo llega) y son necesarios si un hombre desea preservar su casa (que es la razón por la que Egeo tiene a Medea viviendo con él)".

${ }^{24}$ Art. 6.5; 17.6; 18.3. Plutarco minimiza la opinión de Ctesias sobre la muerte de Clearco por influencia de Estatira y en contra de Parisatis, como causa final y definitiva por la que Parisatis, arriesgándose a dar muerte a la mujer legítima del rey y madre de sus hijos, preparó el veneno contra Estatira. 
la de su esposa era por el gran amor y la fuerte confianza existente entre ellos ${ }^{25}$.

La tercera, que por otra parte es la más evidente y concluyente, es que los asesinados son, de una manera u otra, adversarios políticos que se cruzan en el camino del personaje en cuestión: Pirro de Neoptólemo, Teseo de Medea y Estatira de Parisatis. El banquete se presenta entonces como la escena donde se revela una de las caras del poder, el de la violencia que se usa para conservarlo ${ }^{26}$. Ninguno de los personajes de estos banquetes duda en utilizar cualquier método para eliminar a su adversario y, de ese modo, seguir conservando el poder que temían perder o estaban perdiendo.

\section{Bibliografía CITADA}

Aguilar, R., "Pharmakon en Plutarco", in A. G. Nikolaidis (ed.), The Unity of Plutarch's Work. Moralia Themes in the Lives, Features of the Lives in the Moralia, Berlin/New York, 2008, pp. 751-71.

Billault, A., "Plutarque et la scène du banquet", in A. G. Nikolaidis (ed.), The Unity of Plutarch's Work. Moralia Themes in the Lives, Features of the Lives in the Moralia, Berlin/ New York, 2008, pp. 577-90.

Garnsey, P., Food and Society in Classical Antiquity, Cambridge, 1999.

Mossman, J. M., "Plutarch, Pyrrus and Alexander", in P. A. Stadter (ed.), Plutarch and the Historical Tradition, London, 1992, pp. 90-108.

Pellizer, E., "Outlines of a Morphology of Sympotic Entertainment”, in O. Murray (ed.), Sympotika. A Symposium on the Symposion, Oxford, 1994, pp. 177-84.

Titchener, F. B., "Everything to do with Dionysus: Banquets in Plutarch's Lives", in J. G. Montes Cala et al. (eds.), Plutarco, Dioniso y el vino. Actas del VI Simposio Español sobre Plutarco (Cádiz, 14-16 de Mayo, 1998), Madrid, 1999, pp. 491-500.

Walker, H. J., Theseus and Athens, New York, 1995.

\footnotetext{
${ }^{25}$ Art. 19.1.

${ }^{26}$ A. Billault, 2008, p. 578.
} 